\title{
Higher-order assemblies in immune signaling: supramolecular complexes and phase separation
}

\author{
Shiyu Xia ${ }^{1}$ (1), Zhenhang Chen ${ }^{2,3}$, Chen Shen ${ }^{1}$, Tian-Min Fu ${ }^{2,3 \bowtie}$ \\ ${ }^{1}$ Department of Biological Chemistry and Molecular Pharmacology, Harvard Medical School, and Program in Cellular and \\ Molecular Medicine, Boston Children's Hospital, Boston, MA 02115, USA \\ 2 Department of Biological Chemistry and Pharmacology, The Ohio State University, Columbus, OH 43210, USA \\ ${ }^{3}$ The Ohio State University Comprehensive Cancer Center, Columbus, OH 43210, USA \\ $\triangle$ Correspondence: fu.978@osu.edu (T.-M. Fu)
}

Received December 3, 2020 Accepted March 1, 2021

\begin{abstract}
Signaling pathways in innate and adaptive immunity play vital roles in pathogen recognition and the functions of immune cells. Higher-order assemblies have recently emerged as a central principle that governs immune signaling and, by extension, cellular communication in general. There are mainly two types of higherorder assemblies: 1) ordered, solid-like large supramolecular complexes formed by stable and rigid protein-protein interactions, and 2) liquid-like phaseseparated condensates formed by weaker and more dynamic intermolecular interactions. This review covers key examples of both types of higher-order assemblies in major immune pathways. By placing emphasis on the molecular structures of the examples provided, we discuss how their structural organization enables elegant mechanisms of signaling regulation.
\end{abstract}

KEYWORDS higher-order assembly, phase separation, signalosome, cGAS, inflammasome, TCR, BCR, TLR, RLR, TNFR, death domain, immune signaling

\section{INTRODUCTION}

The immune system is typically categorized into innate immunity and adaptive immunity. Innate immunity is the first line of immune defense and recognizes general patterns of pathogen or danger signals to evoke immune defense, while adaptive immunity refers to antigen-specific immune response that involves the activation of $T$ cells and $B$ cells. Both innate immunity and adaptive immunity have evolved intricate signaling networks to defend against pathogens or sterile dangers.

A fundamental question in the field is how immune signaling networks make sensitive response to pathogens or danger signals while avoiding over-activation to induce autoimmune diseases. Recent studies have revealed that higher-order assembly, by which biomolecules cluster into large structures of defined or irregular shapes, provides not only a common theme in innate and adaptive immunity, but also elegant mechanisms of sensitivity control and signal transduction (Yin et al., 2015; Du and Chen, 2018; Courtney et al., 2018).

Formation of higher-order assemblies can be achieved in mainly two ways. First, through stable protein-protein interactions, proteins may form supramolecular complexes with solid-like behaviors and defined shapes. These supramolecular complexes are generally termed signalosomes. Second, phase-separated, more dynamic, and liquid-like droplets can form by weaker multivalent interactions. The process through which such condensation occurs is called liquid-liquid phase separation (LLPS).

Examples of the first type of higher-order assemblies include those formed by members of the death domain (DD) superfamily. Found in many cellular receptors, adaptors, and effectors, DDs are protein-protein-interaction domains that mediate numerous host defense pathways in response to pathogen or danger signals. These pathways include Tolllike receptors (TLRs) signaling, RIG-I-like receptors (RLRs) signaling, inflammasomes signaling, tumor necrosis factor receptors (TNFRs) signaling, as well as $B$ cell receptor (BCR) signaling (Fig. 1). The DD superfamily comprises four subfamilies known as DD, death effector domain (DED), 
caspase recruitment domain (CARD), and Pyrin domain (PYD) (Park et al., 2007a). Despite divergence in their primary sequence, the families share a conserved six-helix bundle structure, with minor variations in the lengths and orientations of the helices. Through oligomerization, DDs can form higher-order assemblies known as signalosomes for signal transduction. For instance, the C-terminal cytoplasmic portion of death receptor Fas contains a DD, which oligomerizes upon Fas ligand (FasL) engagement to interact with the DD of Fas-associated protein with death domain (FADD), thereby passing signals downstream (Wang et al., 2010).

Equally intriguing is the second type of higher-order assemblies mediated by LLPS, which represents a new concept of macromolecular organization in cells (Brangwynne et al., 2009; Banani et al., 2017). A hallmark of cells, in particular eukaryotic cells, is that biochemical reactions are spatially and temporally controlled. One way to achieve such control is that eukaryotic cells have many membraneenclosed organelles to separate reactions from one another.
Another way is the formation of membraneless biomolecular condensates mediated by LLPS. Rapid progress in the field has provided ample examples of physiological processes mediated by LLPS, including growth factors-mediated cell signaling, cytoskeleton assembly, transcription, chromatin organization, pathogenesis of neurodegenerative diseases, and immune response (Molliex et al., 2015; Murakami et al., 2015; Patel et al., 2015; Su et al., 2016; Du and Chen, 2018; Sabari et al., 2018; Case et al., 2019; Gibson et al., 2019; Guo et al., 2019; Huang et al., 2019). A key principle governing LLPS is multivalency, which is enabled by many mechanisms including the presence of multiple modular domains or motifs and intrinsic-disorder regions (IDRs) (Li et al., 2012; Han et al., 2012). Functionally, in addition to regulating the spatial organization of biochemical reactions, LLPS accelerates the reactions by increasing the local concentration of reactants. LLPS also contributes to noise reduction and robust response to true signals. Examples of LLPS that we discuss here are cyclic GMP-AMP synthase (cGAS)-stimulator of interferon genes (STING) signaling in

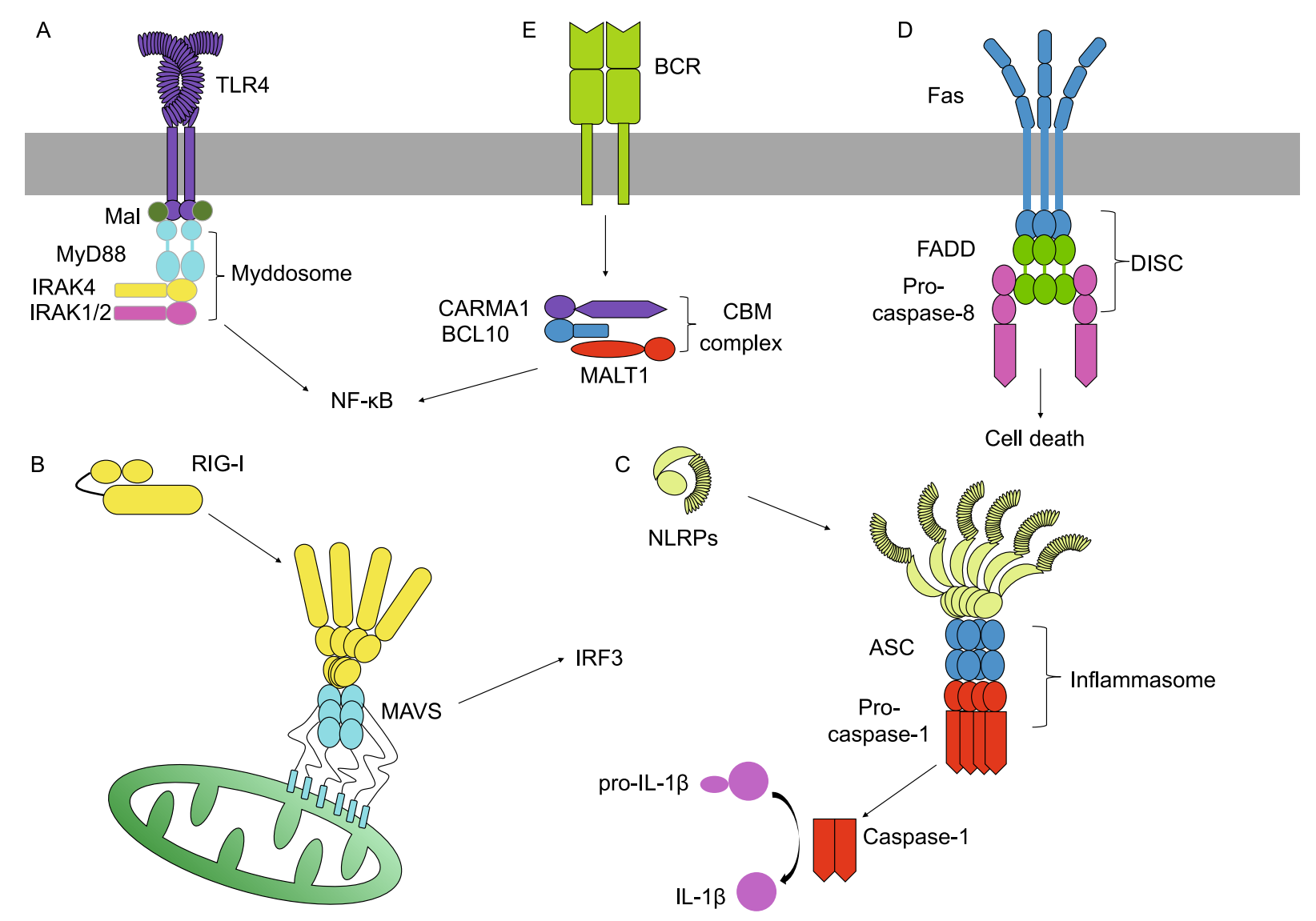

Figure 1. Immune signaling pathways that involve death domain-containing proteins. (A) The myddosome forms downstream of TLR4 via DD-DD interaction. (B) In an antiviral signaling pathway, RIG-I and MAVS assemble into a complex through CARD-CARD interaction. (C) PYD-PYD and CARD-CARD interactions enable the assembly and activation of an ASC-dependent inflammasome. (D) The Fas receptor, a member of the TNFR superfamily, initiates DISC assembly through DD-DD and DED-DED interactions. (E) A B-cell receptor signaling pathway via the CBM signalosome formed by CARD-CARD interaction. 
innate immunity and T-cell receptor (TCR) signaling in adaptive immunity (Su et al., 2016; Du and Chen, 2018).

\section{STRUCTURES AND INTERMOLECULAR INTERACTIONS OF DEATH DOMAINS}

The DD superfamily, including DD, DED, CARD, and PYD, feature a compact six-helix bundle structure arranged in an antiparallel fashion, although the lengths and orientations of the helices can be slightly different (Fig. 2A). First revealed by solution NMR, the structure of the Fas DD shows an amphipathic $\alpha$-helical bundle with hydrophobic residues from each helix lining the core (Huang et al., 1996). Helices a1 and $\alpha 2$ reside at the center whereas $\alpha 3$ through $a 6$ are peripherally located. Later structural studies have unveiled similar six-alpha-helical arrangements in FADD DED (Carrington et al., 2006), caspase-9 CARD (Qin et al., 1999), and apoptosis-associated speck like protein containing a caspase recruitment domain (ASC) PYD (Lu et al., 2014).

During immune response, DDs often assemble into oligomers or polymers through three types of interactions, establishing a molecular basis for protein-protein interaction and signal transduction. The X-ray crystal structure of apoptotic protease activating factor 1 (Apaf-1) CARD in complex with caspase-9 CARD defined Type I interaction, namely the asymmetric interaction between the basic concave surface formed by helices $\alpha 1$ and $\alpha 4$ (Type la surface) and the acidic convex surface formed by helices $\alpha 2$ and $\alpha 3$ (Type Ib surface) (Qin et al., 1999) (Fig. 2B). Later, the crystal structure of Pelle DD in complex with Tube DD defined Type II interaction, mediated mainly by $\alpha 2-\alpha 3$, $\alpha 4-\alpha 5$ loops (Type lla surface) and $\alpha 1-\alpha 2, \alpha 5-\alpha 6$ loops (Type Ilb surface) (Xiao et al., 1999) (Fig. 2C). Besides the 1:1 asymmetric Type I and II interactions revealed by the two dimeric complex structures, the PIDDosome structure uncovered Type III interaction between a3 (Type Illa surface) and $\alpha 1-\alpha 2$ and $\alpha 3-\alpha 4$ loops (Type Illb surface) (Park et al., 2007b) (Fig. 2D). In the PIDDosome structure, all three types of asymmetric interactions together mediate DD oligomerization, which turns out to be a common theme for the DD superfamily and plays crucial roles in cell death and immune signaling. Recently, the cryo-EM revolution enabled highresolution structural determination of several helical filaments formed by members of DD superfamily via Type I, II, and III interactions (Lu et al., 2014). Interestingly, concrete biochemical and structural evidence to date exists only for

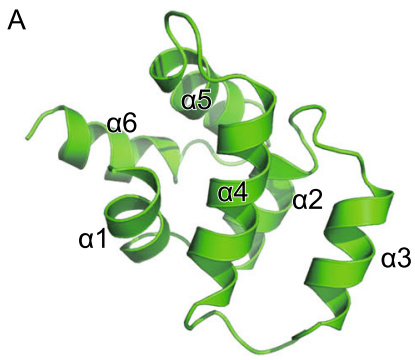

Fas DD

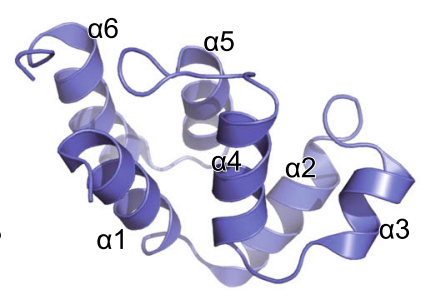

FADD DED

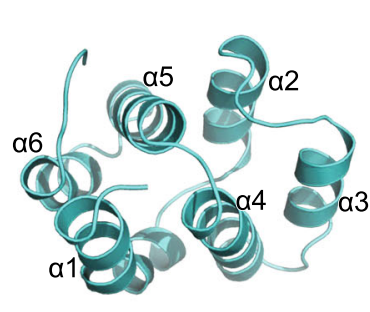

Caspase-9 CARD

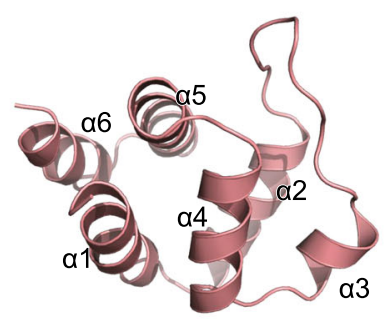

ASC PYD
B

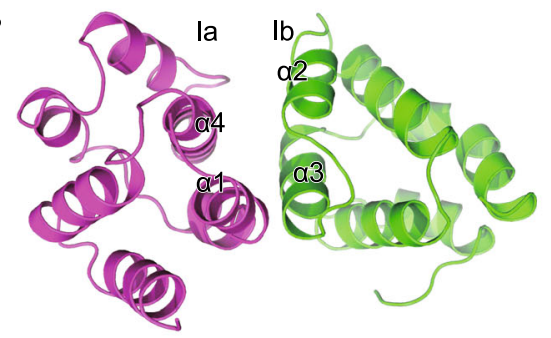

Caspase-9 CARD
Apaf-1 CARD

Type I interaction

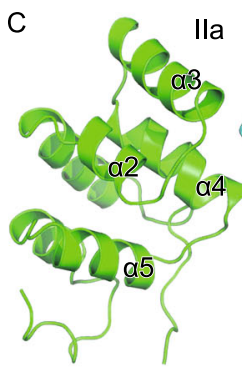

Pelle DD

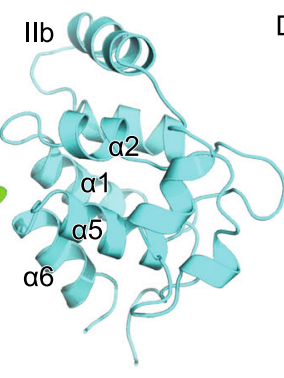

Tube DD
D

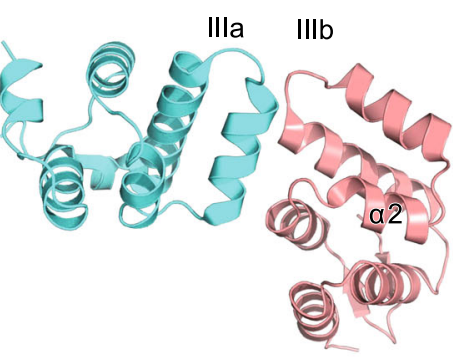

RAIDD DD
PIDD DD

Type II interaction

Type III interaction

Figure 2. Death domain structures and their interactions. (A) Structures of Fas DD (PDB ID: 1DDF), FADD DED (PDB ID: 2GF5), caspase-9 CARD (PDB ID: 3YGS), and ASC PYD (PDB ID: 3J63) show a characteristic six-helix bundle organization conserved in the DD superfamily. (B) Type I interaction revealed by the crystal structure of the Apaf-1 CARD-caspase-9 CARD complex (PDB ID: 3YGS). (C) Type II interaction uncovered by the crystal structure of the Pelle DD-Tube DD complex (PDB ID: 1D2Z). (D) Type III interaction defined by the crystal structure of the RAID DD-PIDD DD complex (PDB ID: 2OF5). 
interactions among DDs of the same subfamilies-for example, between two CARDs or between two PYDs, but not between a CARD and a PYD-although it has been reported that cross interactions may occur (Vajjhala et al., 2015).

As a primer, structural studies have revealed common themes that apply to DD assembly. First, DDs assemble into oligomers with helical symmetry; second, DD assembly is a nucleated hierarchical polymerization process; third, DD assembly is mediated by three different types of proteinprotein interactions; fourth, DD helical oligomers increase the local concentration of kinases or caspases to facilitate their activation. The higher-order assemblies of the DD superfamily represent a new paradigm for signal transduction and a structural basis for threshold responses in immune signaling.

\section{TLR AND RLR SIGNALING}

As a class of pattern recognition receptors (PRRs), TLRs detect a variety of pathogen-associated molecular patterns (PAMPs) outside of the cells or inside cellular compartments, including flagellin, profilin, lipopolysacharrides (LPS), singlestranded RNA (ssRNA), and double-stranded RNA (dsRNA) (Kawai and Akira, 2006). TLRs have N-terminal successive leucine-rich repeats (LRRs) that serve to bind the PAMPs. At their C-termini, there is a Toll/IL-1 receptor (TIR) domain responsible for recruiting cytosolic adapter proteins such as myeloid differentiation factor 88 (MyD88) and MyD88-adapter-like (MAL), which also have a TIR domain (Fig. 3A). In addition to the TIR, MyD88 contains a DD that in turn recruits more downstream proteins such as IL receptor-associated kinase 1/2 (IRAK1/2) and 4 (IRAK4), which contain DDs and kinase domains, via DD-DD interaction (Ferrao et al., 2012). The MyD88-IRAK4-IRAK2 complex assembled by DD interaction is termed a myddosome. Finally, the IRAKs recruit and phosphorylate downstream effector molecules in order to activate NF-KB.

The crystal structure of a myddosome DD complex, consisting of 6 DDs from MyD88, 4 DDs from IRAK4, and 4 DDs from IRAK2, reveals a novel hierarchical and helical assembly mechanism (Lin et al., 2010) (Fig. 3B and 3C). The 14 DDs assemble into a left-handed helical oligomer, with shape and charge complementarity formed at each turn. However, it was unknown how the DDs of MyD88 subunits come into proximity to initiate myddosome assembly. Recently, cryo-EM structures revealed that the TIR domains of TLRs, MAL, and MyD88 polymerize into filaments, which serve as molecular platforms that bring several MyD88 subunits together (Ve et al., 2017). Collectively, these structures provide a mechanistic picture of TLR signaling, where TIR domain-mediated filament formation and DDmediated myddosome assembly co-occur to drive the pathway forward.

RLRs represent another class of PRRs that are located in the cytoplasm and particularly active in the innate immune defense of epithelial and myeloid cells (Loo and Gale, 2011). In contrast to TLRs, which recognize extracellular or endosomal ligands, RLRs such as retinoic acid-inducible gene I (RIG-I) and melanoma differentiation-associated protein 5 (MDA5) recognize viral dsRNA that has entered the cytosol. RIG-I is a sensor of dsRNA with 5' triphosphate (5'-ppp), whereas MDA5 detects long viral dsRNA (Kato et al., 2006; Myong et al., 2009). The structural motif critical for dsRNA binding is a DExD/H box helicase domain at the C-termini of RLRs (Kowalinski et al., 2011; Jiang et al., 2011; Luo et al., 2011; Wu et al., 2013a). In addition to the helicase domain, RIG-I and MDA5 both contain N-terminal tandem CARDs (tCARDs) (Fig. 3D). A model exists that dsRNA binding to the helicase domain causes a conformational change in RIG-I or MDA5 so that the tCARDs are exposed to recruit a downstream adapter by CARD-CARD interaction. The downstream adapter for RLRs is mitochondrial antiviral-signaling protein (MAVS), an N-terminal CARD-containing protein crucial for mediating NF-kB and IRF3 activation (Kawai et al., 2005; Meylan et al., 2005; Seth et al., 2005; Xu et al., 2005). Other than RIG-I and MDA5, a third RLR is LGP2, which shares the helicase domain but does not contain the CARD. Despite a previously assumed inhibitory role due to the lack of the signaling domain CARD, LGP2 was shown to increase the initial rate of MDA5-dsRNA interaction and enhance MDA5-mediated antiviral signaling (Bruns et al., 2014). The molecular mechanism of the cooperation between MDA5 and LGP2 remains elusive.

Similar to the MyD88-IRAK4-IRAK2 myddosome DD complex structure, the crystal structure of RIG-I tCARDs in complex with MAVS CARD and the cryo-EM structure of the MAVS CARD filament support helical assembly as a common mode of signal propagation in both TLR and RLR signaling (Wu et al., 2014) (Fig. 3D and 3E). Zooming in at the end of the filament, RIG-I tCARDs form a tetrameric platform that recruits MAVS CARD by Type I, II, and III interactions, a process commonly referred to as nucleation. Nucleated polymerization into filamentous structures appears as a unified mechanism of DD assembly, which we will revisit in inflammasomes, TNFR signaling, and the CBM signalosome.

\section{INFLAMMASOME SIGNALING AND REGULATION}

Within many types of immune cells, intricate supramolecular complexes termed inflammasomes are activated by diverse microbial and damage-associated signals to trigger immune response. Inflammasome activation ultimately leads to proteolytic activation of the pore-forming protein gasdermin $D$ (GSDMD), release of proinflammatory cytokines such as interkleukin-1 $\beta$ (IL-1 $\beta$ ) and IL-18, and pyroptosis (Xia, 2020; Xia et al., 2020).

There are canonical and noncanonical inflammasomes. Canonical inflammasomes, such as the nucleotide-binding domain, leucine-rich-containing family, PYD-containing protein 3 (NLRP3) inflammasome, the nucleotide-binding 
A

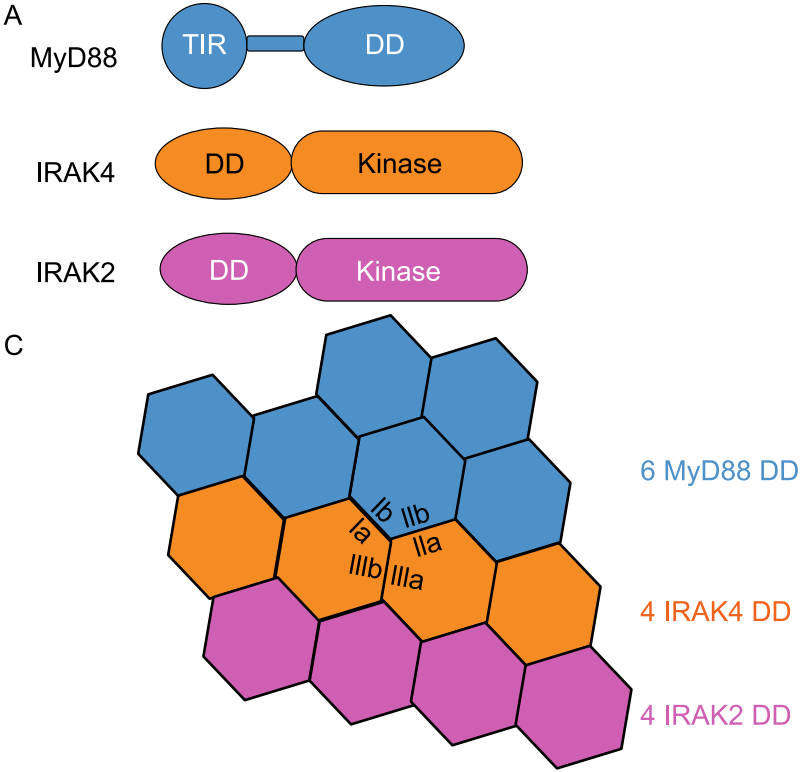

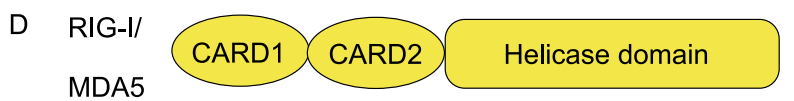
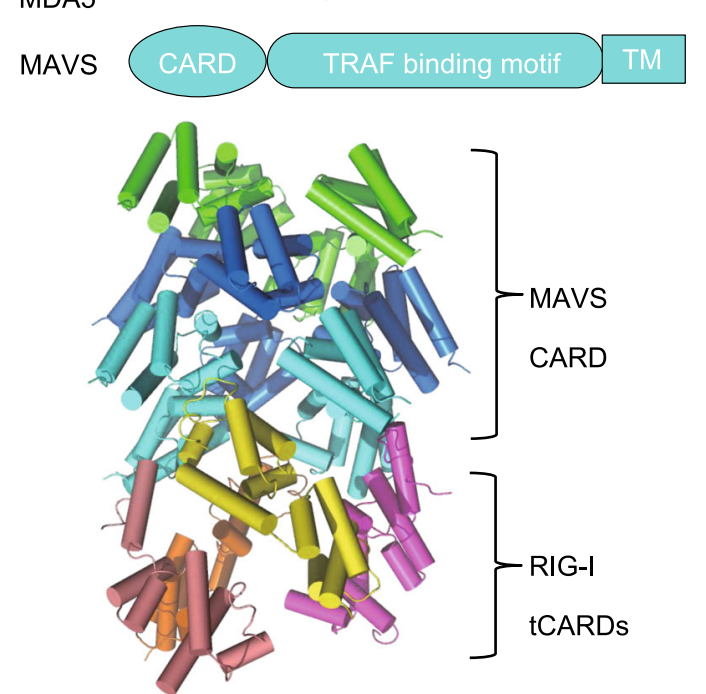

B

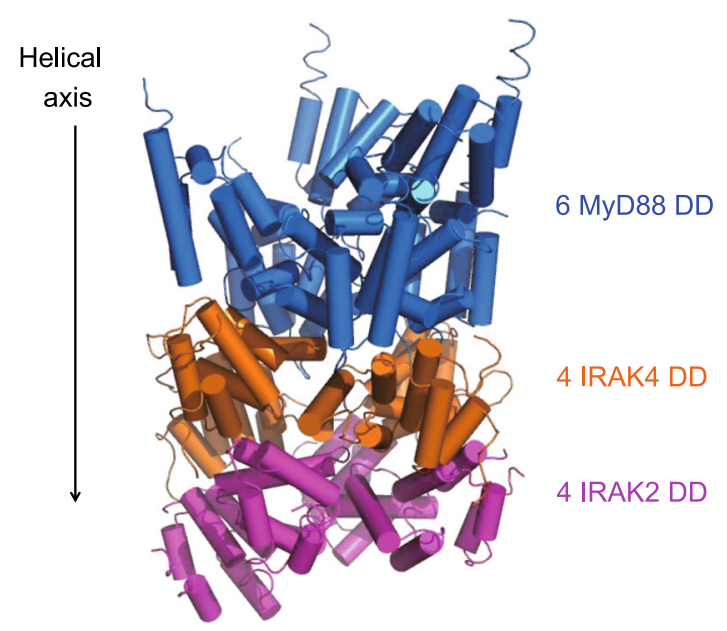

Helical assembly of the myddosome DD complex

E

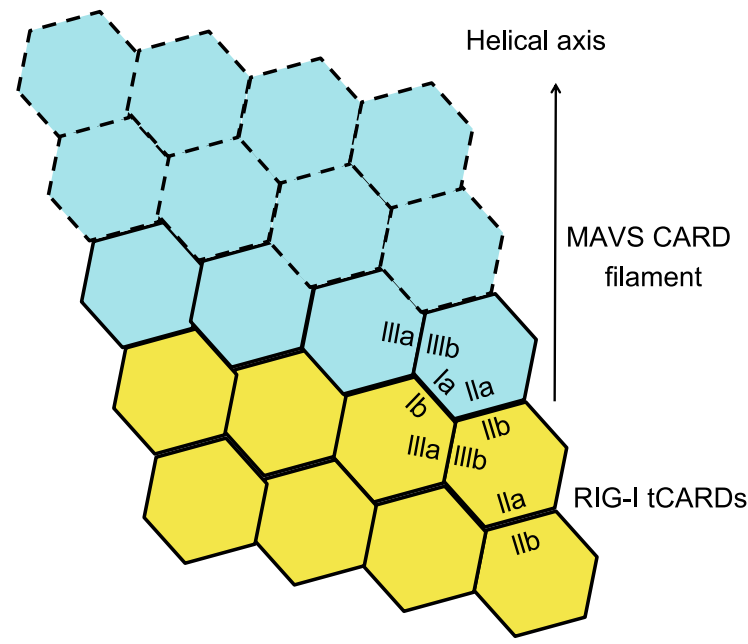

RIG-I nucleates MAVS filament

Figure 3. TLR and RIG-I signaling pathways governed by death domain assembly. (A) Domain architecture of myddosome components MyD88, IRAK4, and IRAK2. (B) Crystal structure of the myddosome DD complex (PDB ID: 3MOP). (C) Helical assembly of the myddosome DD complex with interfaces denoted. (D) Domain architecture of RIG-I, MDA5, and MAVS and the cryo-EM structure of the MAVS CARD-RIG-I tCARDs complex (PDB ID: 4P4H). (E) Helical assembly of the MAVS CARD filament nucleated by RIG-I tCARDs.

domain, leucine-rich-containing family, CARD-containing protein 4 (NLRC4) inflammasome, and the absent in melanoma 2 (AIM2) inflammasome, contain sensors of various signals including flagellin, extracellular ATP, $\mathrm{K}^{+}$efflux, and cytosolic dsDNA (Hornung et al., 2009; Lightfield et al., 2011; Zhao et al., 2011; Munoz-Planillo et al., 2013). While canonical inflammasomes recruit and activate caspase-1, the noncanonical inflammasome relies on direct activation of murine caspase-11 (and human orthologs caspase-4 and -5) by cytosolic LPS (Shi et al., 2014).

Recently, the assembly mechanisms of the three canonical inflammasomes mentioned above have been elucidated by structural and biochemical investigations (Jin et al., 2012; Lu et al., 2014; Diebolder et al., 2015; Hu et al., 2015; Lu et al., 2015; Zhang et al., 2015) (Fig. 4A-D). While the AIM2 and NLRP3 inflammasomes rely on the adapter protein ASC to activate caspase-1, the NLRC4 inflammasome directly 


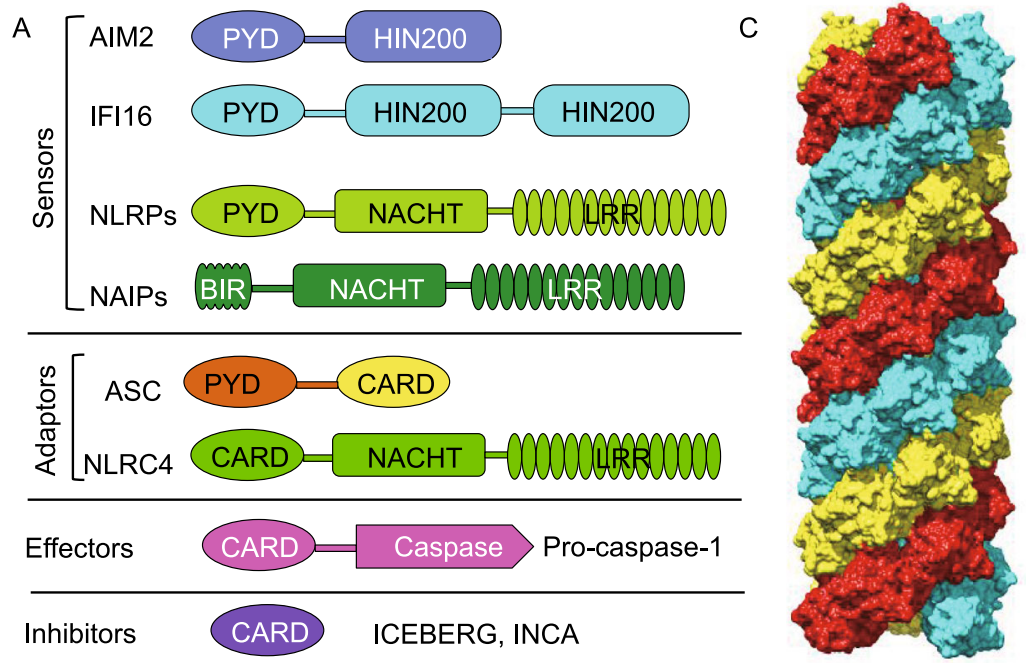

ASC PYD filament

B

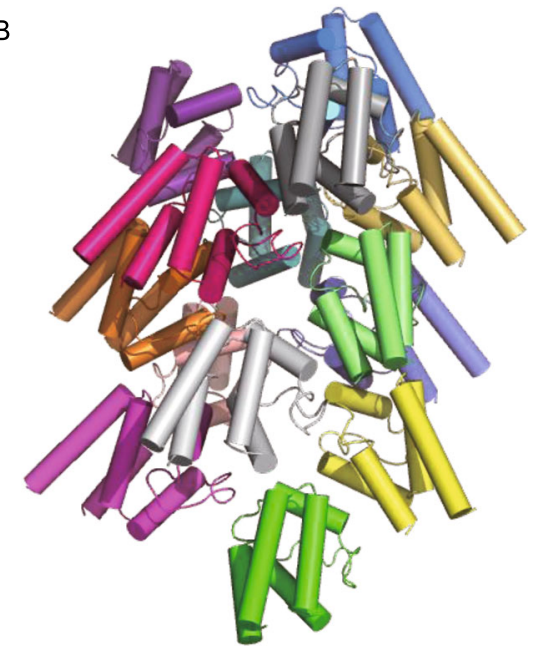

AIM2 PYD filament

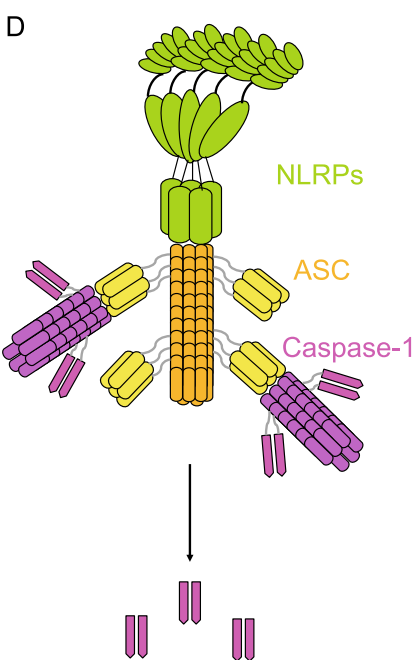

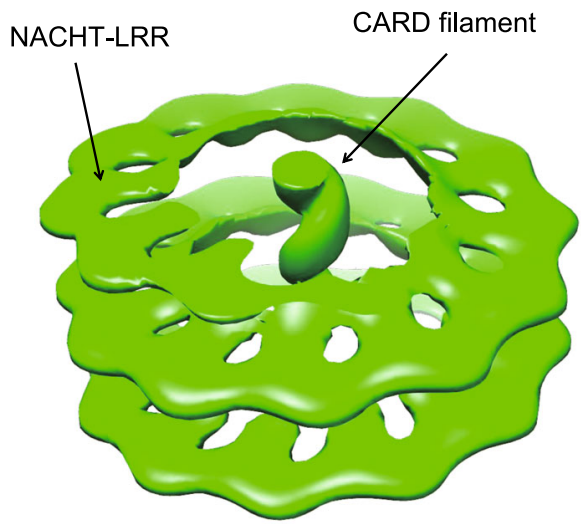

Spiral assembly of the NAIP/NLRC4 inflammasome

E Caspase-1 CARD

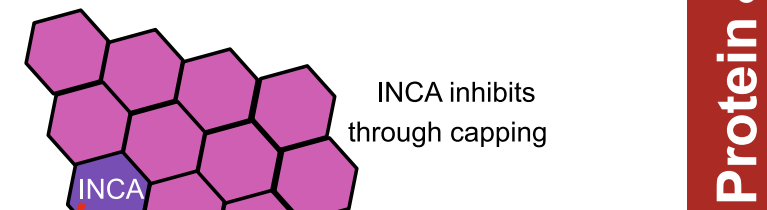

Figure 4. Death domain-mediated inflammasome signaling and regulation. (A) Domain organization of inflammasome components. (B) Cryo-EM structure of the AIM2 PYD filament (PDB ID: 6MB2). (C) Cryo-EM structure of the ASC PYD filament (PDB ID: 3J63) and cryo-electron tomography (cryo-ET) structure (PDB ID: 5AJ2) of the NAIP/NLRC4 inflammasome reveal helical assembly of PYD and CARD. (D) A model of NLRP inflammasome assembly mediated by PYD-PYD interaction between NLRP and ASC and CARD-CARD interaction between ASC and caspase-1. (E) Mechanisms of INCA and ICEBERG as inhibitors of CARD helical assembly during inflammasome activation.

engages and activates caspase-1. The ASC-dependent inflammasomes share a nucleated polymerization mechanism, where AIM2 and NLRP3 serve as nucleators for the polymerization of ASC through PYD-PYD interaction (Fig. 4B and 4C). It should be noted that although AIM2 PYD can assemble into a filament, the oligomerization of NLRP3 PYD is mediated by its NACHT domain (Lu et al., 2014). As an adaptor protein, ASC contains an N-terminal PYD and a C-terminal CARD, which are responsible for interaction with upstream PYD-containing sensors and downstream CARDcontaining effectors (Fig. 4A). Both PYD and CARD of ASC are able to assemble into filaments with helical symmetry (Lu et al., 2014; Li et al., 2018). Different from the ASC-dependent inflammasomes, the helical assembly of the NLRC4 inflammasome is initiated through the activation of the sensor and nucleator NAIP by bacterial ligands (Diebolder et al., 2015; Zhang et al., 2015). In this spiral structure, NLRC4 CARD forms a filament at the center of the spiral, whereas the NACHT and LRR domains assemble into disk-like structures along the CARD filament axis (Fig. 4C). Sharing similar architecture, both ASC and NLRC4 CARD filaments recruit downstream caspase-1 through CARD-CARD 
interaction (Fig. 4D). Polymerization of caspase-1 CARD into filaments locally concentrates caspase- 1 and induces the dimerization and auto-processing of caspase- 1 into its active p10 and p20 fragments, which then cleave pro-IL-1 $\beta$, pro-IL18 , and GSDMD to elicit inflammation and pyroptosis.

Given that cooperativity in nucleated polymerization facilitates rapid filament assembly, inflammasome activation exhibits an all-or-none behavior. It is therefore crucial that cells have evolved elaborate strategies to curb inflammasome activation when necessary. Recent structural studies highlighted two DD filament inhibitors - inhibitor of CARD (INCA) and ICEBERG (Lu et al., 2016). Known as CARDonly proteins (COPs), INCA and ICEBERG both contain one single CARD (Fig. 4A). However, they function by distinct mechanisms (Fig. 4E). ICEBERG interferes with the interaction between caspase-1 CARD and other CARD-containing proteins through commingling. In contrast, INCA prevents caspase-1 CARD polymerization into filaments through a capping mechanism. A closer inspection at subunit oligomerization interfaces reveals that INCA is defective in Type $\mathrm{lb}$ and Type $\mathrm{llb}$, two of the six interfaces required for CARD-CARD interaction and filament formation. Other known inhibitors of DD assembly include PYD-only proteins ASC2 and POP2, albeit unclear molecular mechanisms (Natarajan et al., 2006; Ratsimandresy et al., 2017).

\section{A TNFR PATHWAY}

The TNFR superfamily comprises type-I transmembrane receptors that respond to TNF-like ligands and plays crucial roles in numerous physiological processes including lymphoid development, immunity, cellular homeostasis, and apoptosis. Certain TNFR superfamily members, such as the Fas receptor and TNFR1, contain DDs in their intracellular portions, which can initiate cell death signaling (Fig. 5A). Upon FasL engagement, Fas DD recruits adapter protein FADD, which is composed of a DD and a DED, through DDDD interaction. FADD further recruits caspase-8 through DED-DED interaction to form a ternary complex known as the death-inducing signaling complex (DISC), which facilitates the dimerization and activation of caspase-8.

Crystallographic and cryo-EM studies of the Fas DDFADD DD complex revealed asymmetric oligomers of 5 to 7 Fas DDs and 5 FADD DDs (Wang et al., 2010). In the crystal structure, 5 Fas DDs and 5 FADD DDs form a two-layered structure that resembles the myddosome helical assembly (Fig. 5B). Based on quantitative western blots and mass spectrometry, caspase-8 is about 7- to 9-fold more abundant than FADD at the DISC, an observation that hints at a signal amplification function of DD assembly (Dickens et al., 2012; Schleich et al., 2012). Consistently, caspase-8 also robustly polymerizes into filaments with helical symmetry due to the presence of tandem DEDs (tDEDs) at its $\mathrm{N}$-terminus (Shen et al., 2015; Fu et al., 2016) (Fig. 5C). Similar to other DD assemblies, caspase-8 tDED filaments utilize all three types of interactions (Fig. 5D). However, compared to other filaments formed by single domains, caspase-8 tDED filaments harbor more interactions, such that each type II or type III interface can each engage two different interfaces. The quasi-equivalent interactions in type II and type III interfaces are achieved through charge-charge complementarity, which is critical for the assembly and regulation of caspase-8 tDED filaments. Further structural modeling and biochemical assays showed that DISC assembly is a hierarchical, unidirectional process (Fu et al., 2016).

\section{THE CBM SIGNALOSOME IN BCR SIGNALING}

Downstream of the B-cell receptor, CARM1, BCL10, and MALT1 assemble into a ternary complex known as the CBM signalosome to mediate NF-KB activation. CARMA1 is composed of an $\mathrm{N}$-terminal CARD, a central coiled-coil domain, and a C-terminal MAGUK domain. Upon activation, CARMA1 recruits BCL10 through CARD-CARD interaction, after which the C-terminal Ser/Thr-rich domain of BCL10 interacts with the $\lg 1 / 2$ domain of MALT1 to form the CBM signalosome (Fig. 5E). Together with the crystal structure of CARMA1 CARD, cryo-EM structure of the BCL10 CARD filament and time-lapse confocal microscopy provide insights into the assembly process of the CBM signalosome complex (Qiao et al., 2013; David et al., 2018). Nucleated by CARMA1 CARD, BCL10 CARD unidirectionally polymerizes into filaments through Type I, II, III interactions, a highly unified mechanism of assembly for the DD superfamily.

\section{CGAS-STING SIGNALING}

cGAS is a major cytosolic DNA sensor in innate immunity (Sun et al., 2013). Upon DNA engagement, cGAS produces a second messenger called cGAMP, which binds and activates the downstream effector protein STING (Ablasser et al., 2013; Gao et al., 2013a; Sun et al., 2013; Wu et al., 2013b) (Fig. 6A). Upon activation, STING recruits TANKbinding kinases 1 (TBK1), which then phosphorylates interferon regulatory factor 3 (IRF3), leading to the dimerization and nucleus translocation of IRF3 for the transcriptional induction of type I IFNs and inflammatory cytokines to trigger immune response (Ablasser and Chen, 2019; Zhang et al., 2020) (Fig. 6A). Accumulating evidence has shown that this cGAS-STING signaling pathway plays vital roles in many physiological and pathophysiological processes, including anti-virus immunity, autophagy, cellular senescence, and inflammatory diseases (Li et al., 2013b; Gray et al., 2015; Chen et al., 2016; An et al., 2017; Yang et al., 2017; Gui et al., 2019).

Structural investigation of cGAS and STING has revealed the molecular principles governing the assembly and function of cGAS and STING. cGAS is composed of a disordered $\mathrm{N}$-terminal domain and a C-terminal catalytic domain (Fig. 6B), which belongs to a large family of nucleotidyltransferase (NTases) responsible for catalyzing the transfer of nucleoside monophosphates. The catalytic domain of 
A

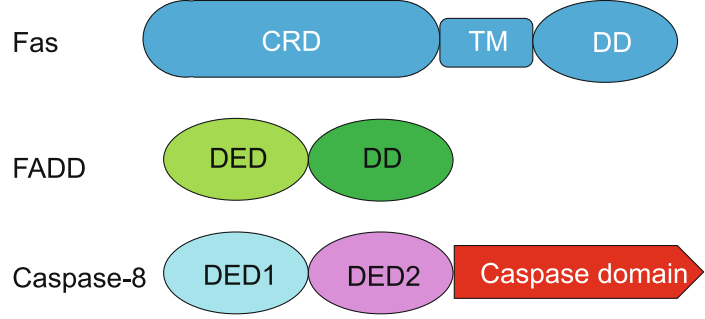

B

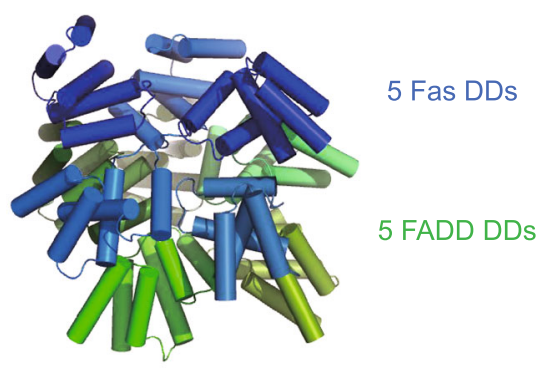

D

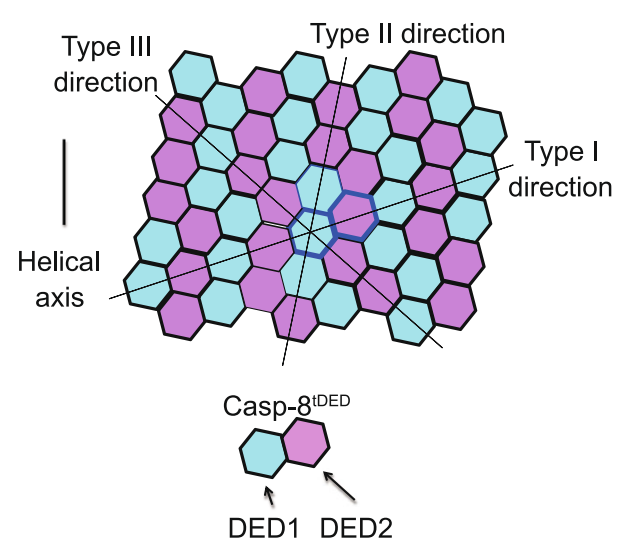

C
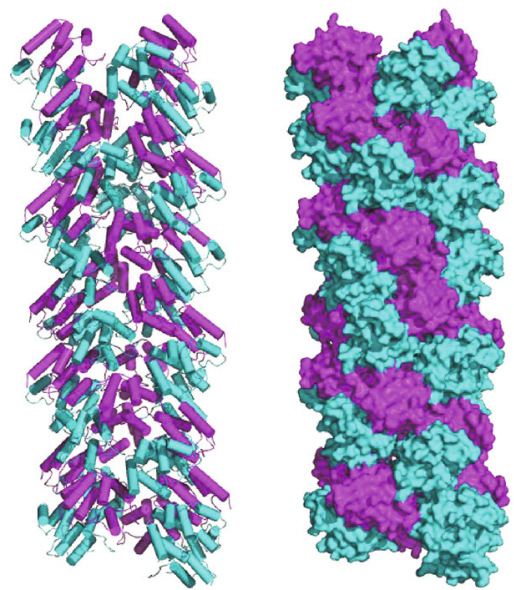

Caspase-8 tDED filament

E CARMA1

CARD CC MAGUK

BCL10

MALT1

CARD S/T rich

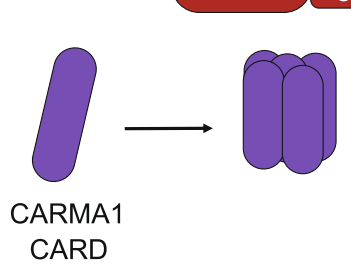

Unidirectional filament growth

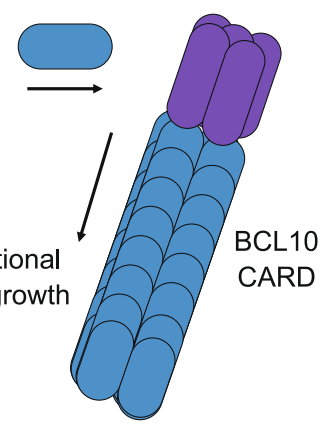

Figure 5. Death domain signaling in Fas and CBM signalosome pathways. (A) Domain architecture of DISC components including receptor Fas, adaptor FADD, and effector caspase-8. (B) Crystal structure of the Fas DD-FADD DD complex (PDB ID: 3OQ9) displays helical assembly similar to the MyD88-IRAK4-IRAK2 DD complex described above. (C) Cryo-EM structure of caspase-8 tDEDs (PDB ID: 5L08) shows helical assembly into filaments. (D) Extensive inter-subunit interactions in caspase-8 tDED filaments. (E) Domain architecture of CBM signalosome components and a nucleated polymerization model of CARMA1 CARDBCL10 CARD filaments.

cGAS adopts a bilobal fold, which contains an N-terminal lobe that is composed of two helices and a twisted beta sheet for catalysis and a C-terminal lobe composed of a helical bundle for mediating DNA binding and dimerization (Gao et al., 2013a; Civril et al., 2013) (Fig. 6B). The large cleft between the two lobes is responsible for binding substrates (Fig. 6B). Opposite to the substrate-binding cleft is a surface groove that can bind DNA (Fig. 6B). cGAS utilizes positively charged residues to engage the sugar-phosphate backbone of DNA, which explains the lack of sequence specificity in DNA recognition by cGAS (Civril et al., 2013; Gao et al., 2013a). DNA binding triggers the re-organization of the cGAS catalytic site to enhance substrate accessibility and thereby promotes the synthesis of cGAMP (Civril et al., 2013; Gao et al., 2013a).

Although DNA ligands shorter than 20 bp are sufficient to trigger CGAMP production, longer DNA is required for the full activation of cGAS in vitro and cGAS signaling in vivo (Luecke et al., 2017). Structures of DNA-bound cGAS showed two DNA-binding sites in CGAS that allowed the formation of a 2:2 DNA-cGAS complex (Li et al., 2013a; Zhang et al., 2013) (Fig. 6C). More strikingly, structures of long DNA ligands in complex with cGAS revealed higherorder assembly of cGAS dimers along the DNA ligands 
A

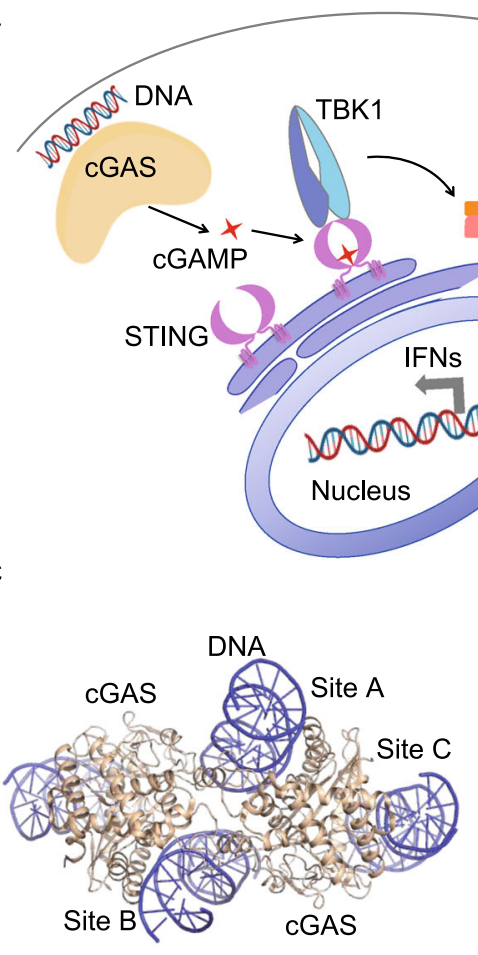

$\mathrm{F}$

STING
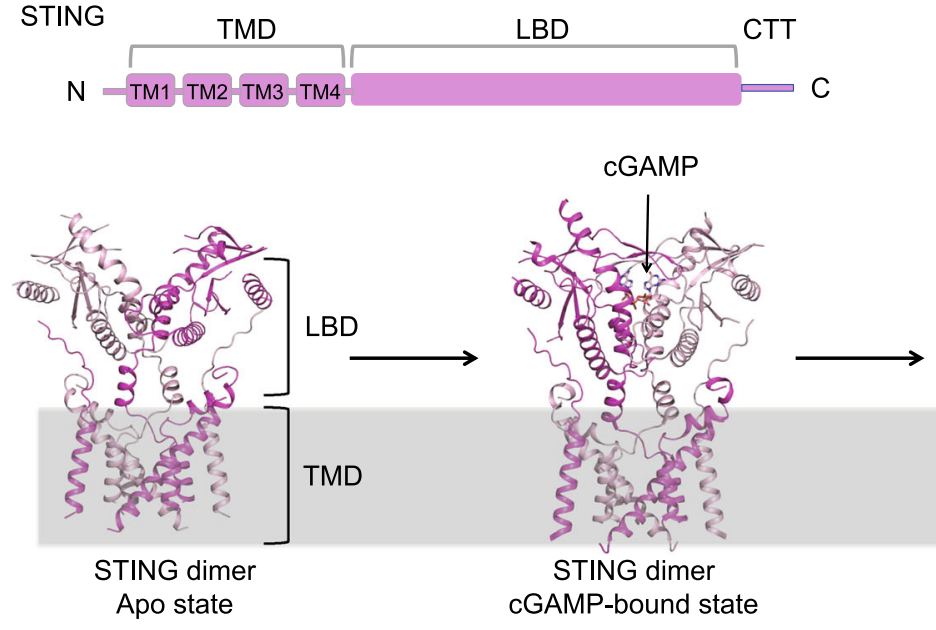

B

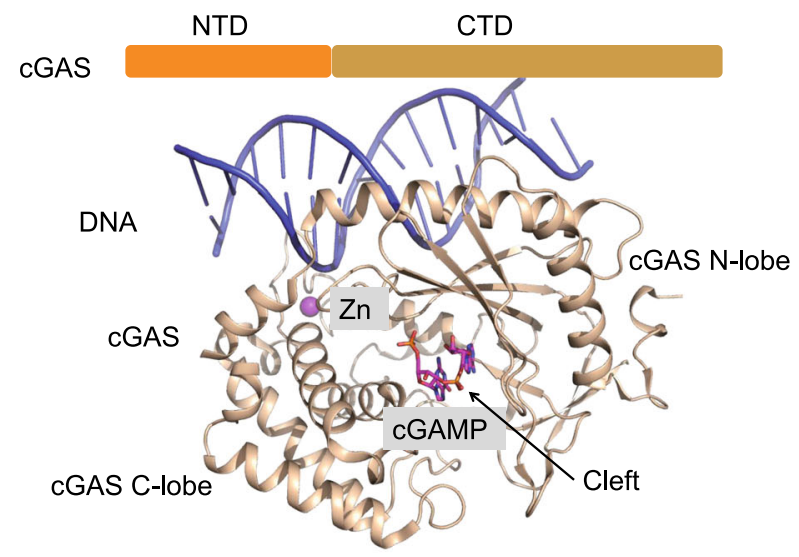

D

IRF3

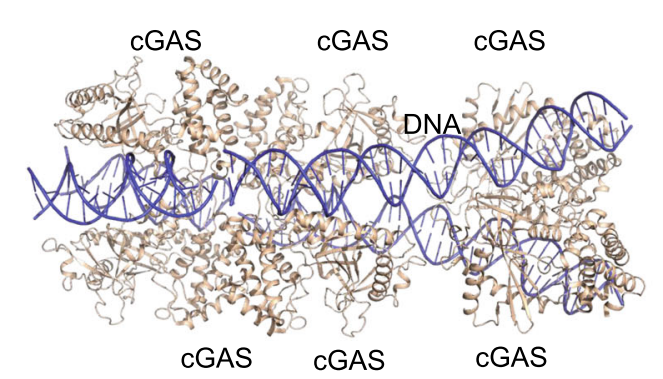

$E$

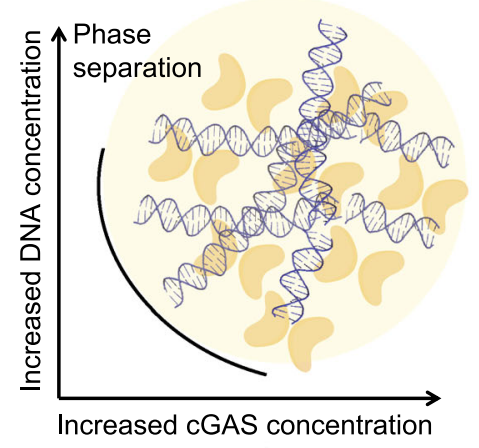

Figure 6. Higher-order assemblies in cGAS-STING signaling. (A) Overview of key molecules in CGAS-STING signaling. (B) Crystal structure of monomeric cGAS in complex with DNA and 2'3'-cGAMP (PDB ID: 4K9A). (C) Crystal structure of dimeric cGAS in complex with DNA (PDB ID: 4LEZ) showing three DNA binding sites in cGAS. Site $A$ and site $B$ contribute to the formation of the 2:2 cGAS-DNA complex. Site $C$ serves as a third interaction site between cGAS and DNA, which is important for phase separation. (D) Crystal structure of oligomeric cGAS bound to long DNA ligands (PDB ID: $5 \mathrm{~N} 6 \mathrm{II}$ ). (E) Liquid-liquid phase separation formed by cGAS and DNA ligands. (F) Mechanisms of CGAMP-induced STING oligomerization and subsequent recruitment and activation of TBK1. STING is composed of an N-terminal transmembrane domain, a ligand-binding domain (LBD), and a C-terminal tail (CTT). Apo STING exists as a constitutive dimer on the ER membrane (PDB ID: 6NT6). Upon cGAMP engagement, dimeric STING undergoes large conformational changes and forms clusters (PDB ID: 6NT7, 6NT8). Activated STING exposes its CTT for the recruitment of TBK1 (PDB ID: 6NT9). The STING oligomer brings many TBK1 molecules into proximity for their transautophosphorylation and activation. 
(Andreeva et al., 2017) (Fig. 6D). The assembly process appears cooperative given that the initial 2:2 cGAS-DNA complex allows rearrangement of the two DNA molecules in a parallel fashion (Andreeva et al., 2017).

More recent findings revealed that long DNA can further induce LLPS by cGAS (Du and Chen, 2018) (Fig. 6E). In cells, LLPS is fundamental for the formation of membraneless cellular compartments, including the stress granules, germ granules, $\mathrm{P}$ bodies, Cajal bodies, nuclear speckles, and the nucleolus (Hyman et al., 2014; Shin and Brangwynne, 2017). Studies have revealed that multivalency of macromolecules is the key factor that drives LLPS, which is also true in the case of cGAS. As mentioned above, cGAS contains a disordered $\mathrm{N}$-terminal domain and a $\mathrm{C}$-terminal catalytic domain, both of which contain DNA-binding sites. The high density of positively charged residues in the $\mathrm{N}$-terminal domain and the three identified DNA-binding sites in the C-terminal domain provide the structural basis for multivalent interaction between cGAS and DNA. Indeed, biochemical assays showed that cGAS and DNA form liquid condensates in a concentration-dependent manner (Du and Chen, 2018). LLPS by cGAS and DNA was shown to enhance the enzymatic activity of cGAS for the synthesis of 2'3'-cGAMP (Du and Chen, 2018). By contrast, a more recent study revealed that cGAS-DNA LLPS does not directly control 2'3'-cGAMP synthesis (Zhou et al., 2021). Instead, cGAS-DNA LLPS serves to resist suppression by multiple negative immune regulators including barrier-to-autointegration factor 1 (BAF1) and the cytosolic exonuclease Trex1 (Zhou et al., 2021). As a major suppressor of DNA sensing, Trex1 degrades cytosolic dsDNA to prevent autoimmune disease (Kavanagh et al., 2008). In this context, cGAS-DNA LLPS protects DNA from degradation by limiting the entry of Trex1 into the cGAS-DNA LLPS as well as suppressing Trex 1 exonuclease activity within the LLPS (Zhou et al., 2021). Together, current studies reveal at least three biological implications for the formation of cGAS-DNA LLPS. First, long DNA is more potent than short DNA in triggering cGAS signaling because long DNA has a higher valency than short DNA and a lower threshold to induce the formation of LLPS. Second, the formation of cGAS-DNA phase separation provides a new mechanism for balancing DNA-dependent immune activation and suppression. Third, phase separation allows the cGAS signaling pathway to mount digital immune defense against infection while avoiding autoimmune reactions to low concentrations of selfDNA. Further study is required to fully discern the functional roles of the cGAS-DNA LLPS in vivo.

As the downstream effector protein of cGAS, STING is located at the endoplasmic reticulum membrane and composed of an $\mathrm{N}$-terminal domain containing four transmembrane helices, a cytosolic ligand-binding domain (LBD) for recognizing cGAMP, and a C-terminal tail (CTT) for recruiting downstream TBK1 (Fig. 6F). Earlier crystal structures showed that the LBD of STING assembles as a constitutive dimer for binding cGAMP (Ouyang et al., 2012; Yin et al.,
2012; Gao et al., 2013b; Zhang et al., 2013). cGAMP engagement induces the rotation of the two LBDs toward each other to form a closed conformation, which is coupled to the activation of STING for downstream signaling. Recently, a cryo-EM study of full-length STING and its complex with cGAMP revealed two unexpected features that are critical for STING activation (Shang et al., 2019) (Fig. 6F). First, upon cGAMP engagement, the LBD of STING undergoes a $180^{\circ}$ rotation relative to the TM domain. Second, a loop on the side of LBD is switched to a less extended conformation for mediating the STING dimer-dimer interaction, leading to the formation of STING oligomers. The C-terminal tails of dimeric STING recruit and activate TBK1. The oligomerization of STING is critical for the activation of TBK1 because the STING oligomers cluster multiple TBK1 dimers to facilitate trans-autophosphorylation of the activation loop, which is a critical step in TBK1 activation (Zhang et al., 2019; Zhao et al., 2019).

\section{TCR SIGNALING}

TCR is responsible for detecting antigens that are presented by major histocompatibility (MHC) molecules. In cells, TCR is composed of $\alpha \beta$ or $\gamma \delta$ heterodimers, CD3 $\varepsilon \delta, C D 3 \varepsilon \gamma$, and a $\zeta$ chain homodimer in a 1:1:1:1 stoichiometry (Courtney et al., 2018) (Fig. 7A). Here we use $\alpha \beta-T C R$ as an example to illustrate the activation mechanism of TCR. In the TCR complex, each $\zeta$ chain has a cytosolic ITAM motif with multiple tyrosine residues, which can be phosphorylated by Lck kinase that is brought to proximity to TCR by CD4 or CD8, which are co-receptors of TCR (Shaw et al., 1990) (Fig. 7A). The phosphorylated motifs within the TCR complex recruit the Zap70 kinase, leading to its phosphorylation and subsequent activation, which initiates TCR signaling (van Oers et al., 1994; Thill et al., 2016) (Fig. 7A).

As T cells require fewer than 10 agonistic $\mathrm{MHC}$ molecules or even only 1 single agonistic MHC molecule to trigger a full response (Irvine et al., 2002), it is clear that signal amplification plays a critical role in T cell activation. A key mechanism for signal amplification is provided by the LAT (linker for activation of T-cells) signaling hub. Activated Zap70 phosphorylates the adaptor protein LAT for the recruitment of additional adaptor proteins such as Grb2 and GADS, which will further engage SOS or SLP-76 (Courtney et al., 2018) (Fig. 7A). These proteins in the LAT signaling hub will activate downstream effector proteins which mediate mitogenactivated protein kinase (MAPK) activation and actin polymerization (Fig. 7A). Recent studies by in vitro reconstitution using supported lipid bilayers revealed that the LAT and downstream signaling proteins assemble into condensates by LLPS (Huang et al., 2016; Su et al., 2016) (Fig. 7B). The process involves multivalent interactions that are mediated by LAT, Grb2, and SOS (Su et al., 2016) (Fig. 7B). The micrometer-sized clusters of LAT and the associated proteins serve as a biochemical reaction center that facilitates MAPK activation and actin polymerization. 


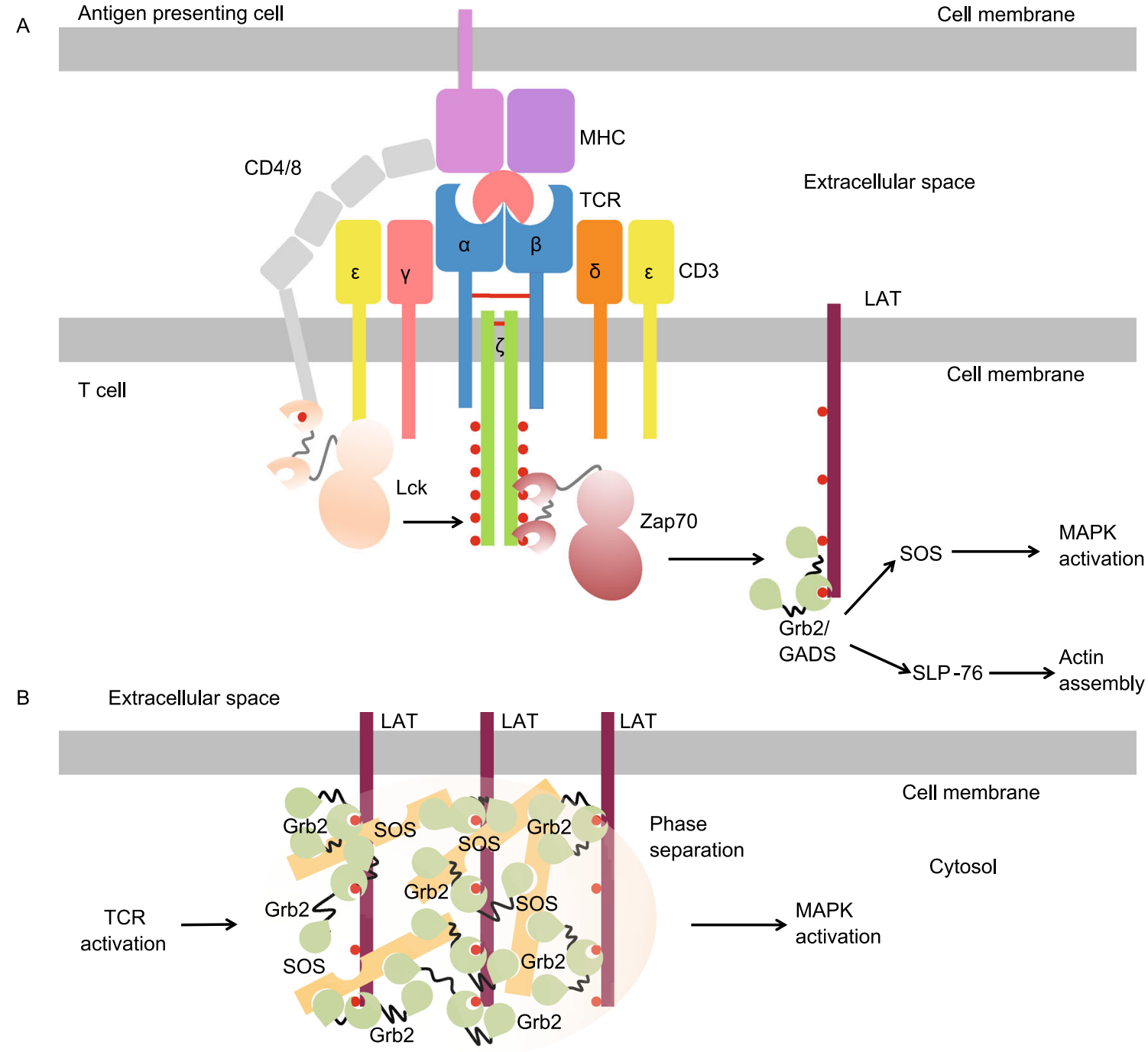

Figure 7. Phase separation in TCR signaling. (A) Overview of the TCR signaling pathway. (B) Schematic of liquid-liquid phase separation that is assembled by LAT, Grb2, and SOS.

\section{CONCLUDING REMARKS}

Higher-order assemblies, in the forms of solid-like supramolecular complexes and phase-separated liquid droplets, represent a new paradigm for cell signaling. Different from the classical model of cell signaling that is triggered by conformational changes of receptors, higher-order assembly generally involves the polymerization of receptors, adaptors, and effectors into large structures of defined rigid shapes or into dynamic liquid-like condensates. These two types of higher-order assemblies share many features, such as concentration dependence, multivalency, as well as nucleated polymerization (Wu and Fuxreiter, 2016). A key difference between these two types of higher-order assemblies is that the solid-like supramolecular complexes are often stable and feature a unidirectional assembly process, while the liquid condensates are more dynamic and reversible. In some cases, liquid-like condensates can also convert into the more stable and solid-like state (Molliex et al., 2015; Murakami et al., 2015; Patel et al., 2015). It is likely that biological systems have evolved these two different types of higher-order assemblies to fine-tune the dynamics and reversibility of physiological processes.

Signaling by higher-order assemblies offers at least two advantages. First, the cooperative assembly process allows cells to make digital, threshold-like response. In other words, cells utilize this elegant mechanism to filter out biological noise and only respond to real signals that are strong and persistent. As immune signaling is a double-edged sword that may result in defense or autoimmunity, the immune system has evolved such an elegant mechanism that utilizes higher-order assembly to prevent unnecessary immune activation. Second, higher-order assemblies create a biochemical reaction center that significantly increases the local concentration of effector proteins, thereby enabling the 
proximity-driven protein activation and spatial regulation of cell signaling. These advantages may also manifest in cells that do not possess immune functions. We look forward to future discoveries that extend higher-order assemblies to a general principle in cell biology.

\section{ABBREVIATIONS}

AIM2, absent in melanoma 2; Apaf-1, apoptotic protease activating factor 1; ASC, apoptosis-associated speck like protein containing a caspase recruitment domain; BAF1, barrier-to-autointegration factor 1; BCR, B cell receptor; CARD, caspase recruitment domain; COPs, CARD-only proteins; CTT, C-terminal tail; DD, death domain; cyclic GMP-AMP synthase (cGAS); DED, death effector domain; dsRNA, double-stranded RNA; FADD, Fas-associated protein with death domain; FasL, Fas ligand; GSDMD, gasdermin D; IDRs, intrinsicdisorder regions; IL-1 $\beta$, interkleukin-1 $\beta$; INCA, inhibitor of CARD; IRAK, IL receptor-associated kinase; IRF3, interferon regulatory factor 3; LAT, linker for activation of T-cells; LLPS, liquid-liquid phase separation; LPS, lipopolysacharrides; LRRs, leucine-rich repeats; MAL, MyD88-adapter-like; MAPK, mitogen-activated protein kinase; MAVS, mitochondrial antiviral-signaling protein; MDA5, melanoma differentiation-associated protein 5; MHC, major histocompatibility; MyD88, myeloid differentiation factor 88; NLRC4, the nucleotidebinding domain leucine-rich-containing family CARD-containing protein 4; NLRP3, the nucleotide-binding domain leucine-richcontaining family PYD-containing protein 3; NTases, nucleotidyltransferase; PAMPs, pathogen-associated molecular patterns; PRRs, pattern recognition receptors; PYD, pyrin domain; RIG-I, retinoic acid-inducible gene I; RLRs, RIG-l-like receptors; stimulator of interferon genes (STING); ssRNA, single-stranded RNA; STING, stimulator of interferon genes; TBK1, TANK-binding kinases 1; tCARDs, tandem CARDs; TCR, T-cell receptor; tDEDs, tandem DEDs; TIR, Toll/IL-1 receptor; TLRs, Toll-like receptors; TNFRs, tumor necrosis factor receptors.

\section{ACKNOWLEDGEMENTS}

T.M.F. is supported by the Ohio State University Startup fund and C. $\mathrm{S}$. is supported by a Cancer Research Institute Irvington Postdoctoral Fellowship. We apologize for incomplete citations due to space limitations.

\section{DECLARATIONS}

\section{FUNDING}

As stated in above, T.M.F. is supported by the Ohio State University Startup fund and C.S. is supported by a Cancer Research Institute Irvington Postdoctoral Fellowship.

\section{CONFLICTS OF INTEREST}

The authors declare no competing interests.

\section{ETHICS APPROVAL}

This article does not contain any studies with human or animal subjects performed by any of the authors.

\section{CONSENT TO PARTICIPATE}

All the authors participate in the conception and writing of the manuscript.

\section{CONSENT FOR PUBLICATION}

All the authors agree to publish the manuscript.

\section{OPEN ACCESS}

This article is licensed under a Creative Commons Attribution 4.0 International License, which permits use, sharing, adaptation, distribution and reproduction in any medium or format, as long as you give appropriate credit to the original author(s) and the source, provide a link to the Creative Commons licence, and indicate if changes were made. The images or other third party material in this article are included in the article's Creative Commons licence, unless indicated otherwise in a credit line to the material. If material is not included in the article's Creative Commons licence and your intended use is not permitted by statutory regulation or exceeds the permitted use, you will need to obtain permission directly from the copyright holder. To view a copy of this licence, visit http:// creativecommons.org/licenses/by/4.0/.

\section{REFERENCES}

Ablasser A, Chen ZJ (2019) cGAS in action: expanding roles in immunity and inflammation. Science 363:eaat8657

Ablasser A, Goldeck M, Cavlar T, Deimling T, Witte G, Rohl I, Hopfner KP, Ludwig J, Hornung V (2013) cGAS produces a 2'-5'linked cyclic dinucleotide second messenger that activates STING. Nature 498:380-384

An J, Durcan L, Karr RM, Briggs TA, Rice GI, Teal TH, Woodward JJ, Elkon KB (2017) Expression of cyclic GMP-AMP synthase in patients with systemic lupus erythematosus. Arthritis Rheumatol 69:800-807

Andreeva L, Hiller B, Kostrewa D, Lassig C, De Oliveira Mann CC, Jan Drexler D, Maiser A, Gaidt M, Leonhardt $H$, Hornung $V$ et al (2017) cGAS senses long and HMGB/TFAM-bound U-turn DNA by forming protein-DNA ladders. Nature 549:394-398

Banani SF, Lee HO, Hyman AA, Rosen MK (2017) Biomolecular condensates: organizers of cellular biochemistry. Nat Rev Mol Cell Biol 18:285-298

Brangwynne CP, Eckmann CR, Courson DS, Rybarska A, Hoege C, Gharakhani J, Julicher F, Hyman AA (2009) Germline P granules are liquid droplets that localize by controlled dissolution/condensation. Science 324:1729-1732

Bruns AM, Leser GP, Lamb RA, Horvath CM (2014) The innate immune sensor LGP2 activates antiviral signaling by regulating MDA5-RNA interaction and filament assembly. Mol Cell 55:771781

Carrington PE, Sandu C, Wei Y, Hill JM, Morisawa G, Huang T, Gavathiotis E, Wei Y, Werner MH (2006) The structure of FADD 
and its mode of interaction with procaspase-8. Mol Cell 22:599_ 610

Case LB, Zhang X, Ditlev JA, Rosen MK (2019) Stoichiometry controls activity of phase-separated clusters of actin signaling proteins. Science 363:1093-1097

Chen Q, Sun L, Chen ZJ (2016) Regulation and function of the cGAS-STING pathway of cytosolic DNA sensing. Nat Immunol 17:1142-1149

Civril F, Deimling T, De Oliveira Mann CC, Ablasser A, Moldt M, Witte G, Hornung V, Hopfner KP (2013) Structural mechanism of cytosolic DNA sensing by cGAS. Nature 498:332-337

Courtney AH, Lo WL, Weiss A (2018) TCR signaling: mechanisms of initiation and propagation. Trends Biochem Sci 43:108-123

David L, Li Y, Ma J, Garner E, Zhang X, Wu H (2018) Assembly mechanism of the CARMA1-BCL10-MALT1-TRAF6 signalosome. Proc Natl Acad Sci USA 115:1499-1504

Dickens LS, Boyd RS, Jukes-Jones R, Hughes MA, Robinson GL, Fairall L, Schwabe JW, Cain K, Macfarlane M (2012) A death effector domain chain DISC model reveals a crucial role for caspase-8 chain assembly in mediating apoptotic cell death. Mol Cell 47:291-305

Diebolder CA, Halff EF, Koster AJ, Huizinga EG, Koning RI (2015) Cryoelectron tomography of the NAIP5/NLRC4 inflammasome: implications for NLR activation. Structure 23:2349-2357

Du M, Chen ZJ (2018) DNA-induced liquid phase condensation of cGAS activates innate immune signaling. Science 361:704-709

Ferrao R, Li J, Bergamin E, Wu H (2012) Structural insights into the assembly of large oligomeric signalosomes in the Toll-like receptor-interleukin-1 receptor superfamily. Sci Signal 5:re3

Fu TM, Li Y, Lu A, Li Z, Vajjhala PR, Cruz AC, Srivastava DB, Dimaio F, Penczek PA, Siegel RM et al (2016) Cryo-EM structure of caspase-8 tandem DED filament reveals assembly and regulation mechanisms of the death-inducing signaling complex. Mol Cell 64:236-250

Gao P, Ascano M, Wu Y, Barchet W, Gaffney BL, Zillinger T, Serganov AA, Liu Y, Jones RA, Hartmann G et al (2013a) Cyclic $\left[G\left(2^{\prime}, 5^{\prime}\right) p A\left(3^{\prime}, 5^{\prime}\right) p\right]$ is the metazoan second messenger produced by DNA-activated cyclic GMP-AMP synthase. Cell 153:10941107

Gao P, Ascano M, Zillinger T, Wang W, Dai P, Serganov AA, Gaffney BL, Shuman S, Jones RA, Deng $L$ et al (2013b) Structurefunction analysis of STING activation by $\mathrm{c}\left[\mathrm{G}\left(2^{\prime}, 5^{\prime}\right) \mathrm{pA}\left(3^{\prime}, 5^{\prime}\right) \mathrm{p}\right]$ and targeting by antiviral DMXAA. Cell 154:748-762

Gibson BA, Doolittle LK, Schneider MWG, Jensen LE, Gamarra N, Henry L, Gerlich DW, Redding S, Rosen MK (2019) Organization of chromatin by intrinsic and regulated phase separation. Cell 179(470-484):

Gray EE, Treuting PM, Woodward JJ, Stetson DB (2015) Cutting edge: cGAS is required for lethal autoimmune disease in the Trex1-deficient mouse model of Aicardi-Goutieres syndrome. J Immunol 195:1939-1943

Gui X, Yang H, Li T, Tan X, Shi P, Li M, Du F, Chen ZJ (2019) Autophagy induction via STING trafficking is a primordial function of the cGAS pathway. Nature 567:262-266

Guo YE, Manteiga JC, Henninger JE, Sabari BR, Dall'Agnese A, Hannett NM, Spille JH, Afeyan LK, Zamudio AV, Shrinivas K et al
(2019) Pol II phosphorylation regulates a switch between transcriptional and splicing condensates. Nature 572:543-548

Han TW, Kato M, Xie S, Wu LC, Mirzaei H, Pei J, Chen M, Xie Y, Allen J, Xiao $G$ et al (2012) Cell-free formation of RNA granules: bound RNAs identify features and components of cellular assemblies. Cell 149:768-779

Hornung V, Ablasser A, Charrel-Dennis M, Bauernfeind F, Horvath G, Caffrey DR, Latz E, Fitzgerald KA (2009) AIM2 recognizes cytosolic dsDNA and forms a caspase-1-activating inflammasome with ASC. Nature 458:514-518

Hu Z, Zhou Q, Zhang C, Fan S, Cheng W, Zhao Y, Shao F, Wang HW, Sui SF, Chai J (2015) Structural and biochemical basis for induced self-propagation of NLRC4. Science 350:399-404

Huang B, Eberstadt M, Olejniczak ET, Meadows RP, Fesik SW (1996) NMR structure and mutagenesis of the Fas (APO-1/CD95) death domain. Nature 384:638-641

Huang WY, Yan Q, Lin WC, Chung JK, Hansen SD, Christensen SM, Tu HL, Kuriyan J, Groves JT (2016) Phosphotyrosine-mediated LAT assembly on membranes drives kinetic bifurcation in recruitment dynamics of the Ras activator SOS. Proc Natl Acad Sci USA 113:8218-8223

Huang WYC, Alvarez S, Kondo Y, Lee YK, Chung JK, Lam HYM, Biswas KH, Kuriyan J, Groves JT (2019) A molecular assembly phase transition and kinetic proofreading modulate Ras activation by SOS. Science 363:1098-1103

Hyman AA, Weber CA, Julicher F (2014) Liquid-liquid phase separation in biology. Annu Rev Cell Dev Biol 30:39-58

Irvine DJ, Purbhoo MA, Krogsgaard M, Davis MM (2002) Direct observation of ligand recognition by T cells. Nature 419:845-849

Jiang F, Ramanathan A, Miller MT, Tang GQ, Gale M Jr., Patel SS, Marcotrigiano J (2011) Structural basis of RNA recognition and activation by innate immune receptor RIG-I. Nature 479:423-427

Jin T, Perry A, Jiang J, Smith P, Curry JA, Unterholzner L, Jiang Z, Horvath G, Rathinam VA, Johnstone RW et al (2012) Structures of the HIN domain:DNA complexes reveal ligand binding and activation mechanisms of the AIM2 inflammasome and IFI16 receptor. Immunity 36:561-571

Kato H, Takeuchi O, Sato S, Yoneyama M, Yamamoto M, Matsui K, Uematsu S, Jung A, Kawai T, Ishii KJ et al (2006) Differential roles of MDA5 and RIG-I helicases in the recognition of RNA viruses. Nature 441:101-105

Kavanagh D, Spitzer D, Kothari PH, Shaikh A, Liszewski MK, Richards A, Atkinson JP (2008) New roles for the major human 3'-5' exonuclease TREX1 in human disease. Cell Cycle 7:17181725

Kawai T, Akira S (2006) TLR signaling. Cell Death Differ 13:816-825 Kawai T, Takahashi K, Sato S, Coban C, Kumar H, Kato H, Ishii KJ, Takeuchi O, Akira S (2005) IPS-1, an adaptor triggering RIG-Iand Mda5-mediated type I interferon induction. Nat Immunol 6:981-988

Kowalinski E, Lunardi T, McCarthy AA, Louber J, Brunel J, Grigorov B, Gerlier D, Cusack S (2011) Structural basis for the activation of innate immune pattern-recognition receptor RIG-I by viral RNA. Cell 147:423-435

Li P, Banjade S, Cheng HC, Kim S, Chen B, Guo L, Llaguno M, Hollingsworth JV, King DS, Banani SF et al (2012) Phase 
transitions in the assembly of multivalent signalling proteins. Nature 483:336-340

Li X, Shu C, Yi G, Chaton CT, Shelton CL, Diao J, Zuo X, Kao CC, Herr AB, Li P (2013a) Cyclic GMP-AMP synthase is activated by double-stranded DNA-induced oligomerization. Immunity 39:1019-1031

Li XD, Wu J, Gao D, Wang H, Sun L, Chen ZJ (2013b) Pivotal roles of cGAS-cGAMP signaling in antiviral defense and immune adjuvant effects. Science 341:1390-1394

Li Y, Fu TM, Lu A, Witt K, Ruan J, Shen C, Wu H (2018) Cryo-EM structures of ASC and NLRC4 CARD filaments reveal a unified mechanism of nucleation and activation of caspase-1. Proc Natl Acad Sci USA. 115:10845-10852

Lightfield KL, Persson J, Trinidad NJ, Brubaker SW, Kofoed EM, Sauer JD, Dunipace EA, Warren SE, Miao EA, Vance RE (2011) Differential requirements for NAIP5 in activation of the NLRC4 inflammasome. Infect Immun 79:1606-1614

Lin SC, Lo YC, Wu H (2010) Helical assembly in the MyD88-IRAK4IRAK2 complex in TLR/IL-1R signalling. Nature 465:885-890

Loo YM, Gale M Jr. (2011) Immune signaling by RIG-I-like receptors. Immunity 34:680-692

Lu A, Magupalli VG, Ruan J, Yin Q, Atianand MK, Vos MR, Schroder GF, Fitzgerald KA, Wu H, Egelman EH (2014) Unified polymerization mechanism for the assembly of ASC-dependent inflammasomes. Cell 156:1193-1206

Lu A, Li Y, Yin Q, Ruan J, Yu X, Egelman E, Wu H (2015) Plasticity in PYD assembly revealed by cryo-EM structure of the PYD filament of AIM2. Cell Discov 1:1-14

Lu A, Li Y, Schmidt FI, Yin Q, Chen S, Fu TM, Tong AB, Ploegh HL, Mao Y, Wu H (2016) Molecular basis of caspase-1 polymerization and its inhibition by a new capping mechanism. Nat Struct Mol Biol 23:416-425

Luecke S, Holleufer A, Christensen $M H$, Jonsson KL, Boni GA, Sorensen LK, Johannsen M, Jakobsen MR, Hartmann R, Paludan SR (2017) cGAS is activated by DNA in a lengthdependent manner. EMBO Rep 18:1707-1715

Luo D, Ding SC, Vela A, Kohlway A, Lindenbach BD, Pyle AM (2011) Structural insights into RNA recognition by RIG-I. Cell 147:409422

Meylan E, Curran J, Hofmann K, Moradpour D, Binder M, Bartenschlager R, Tschopp J (2005) Cardif is an adaptor protein in the RIG-I antiviral pathway and is targeted by hepatitis $C$ virus. Nature 437:1167-1172

Molliex A, Temirov J, Lee J, Coughlin M, Kanagaraj AP, Kim HJ, Mittag T, Taylor JP (2015) Phase separation by low complexity domains promotes stress granule assembly and drives pathological fibrillization. Cell 163:123-133

Munoz-Planillo R, Kuffa P, Martinez-Colon G, Smith BL, Rajendiran TM, Nunez G (2013) K(+) efflux is the common trigger of NLRP3 inflammasome activation by bacterial toxins and particulate matter. Immunity 38:1142-1153

Murakami T, Qamar S, Lin JQ, Schierle GS, Rees E, Miyashita A, Costa AR, Dodd RB, Chan FT, Michel CH et al (2015) ALS/FTD mutation-induced phase transition of FUS liquid droplets and reversible hydrogels into irreversible hydrogels impairs RNP granule function. Neuron 88:678-690
Myong S, Cui S, Cornish PV, Kirchhofer A, Gack MU, Jung JU, Hopfner KP, Ha T (2009) Cytosolic viral sensor RIG-I is a 5'triphosphate-dependent translocase on double-stranded RNA. Science 323:1070-1074

Natarajan A, Ghose R, Hill JM (2006) Structure and dynamics of ASC2, a pyrin domain-only protein that regulates inflammatory signaling. J Biol Chem 281:31863-31875

Ouyang S, Song X, Wang Y, Ru H, Shaw N, Jiang Y, Niu F, Zhu Y, Qiu W, Parvatiyar K et al (2012) Structural analysis of the STING adaptor protein reveals a hydrophobic dimer interface and mode of cyclic di-GMP binding. Immunity 36:1073-1086

Park HH, Lo YC, Lin SC, Wang L, Yang JK, Wu H (2007a) The death domain superfamily in intracellular signaling of apoptosis and inflammation. Annu Rev Immunol 25:561-586

Park HH, Logette E, Raunser S, Cuenin S, Walz T, Tschopp J, Wu H (2007b) Death domain assembly mechanism revealed by crystal structure of the oligomeric PIDDosome core complex. Cell 128:533-546

Patel A, Lee HO, Jawerth L, Maharana S, Jahnel M, Hein MY, Stoynov S, Mahamid J, Saha S, Franzmann TM et al (2015) A liquid-to-solid phase transition of the ALS protein FUS accelerated by disease mutation. Cell 162:1066-1077

Qiao Q, Yang C, Zheng C, Fontan L, David L, Yu X, Bracken C, Rosen M, Melnick A, Egelman EH et al (2013) Structural architecture of the CARMA1/Bcl10/MALT1 signalosome: nucleation-induced filamentous assembly. Mol Cell 51:766-779

Qin H, Srinivasula SM, Wu G, Fernandes-Alnemri T, Alnemri ES, Shi $Y$ (1999) Structural basis of procaspase-9 recruitment by the apoptotic protease-activating factor 1. Nature 399:549-557

Ratsimandresy RA, Chu LH, Khare S, de Almeida L, Gangopadhyay A, Indramohan M, Misharin AV, Greaves DR, Perlman H, Dorfleutner A et al (2017) The PYRIN domain-only protein POP2 inhibits inflammasome priming and activation. Nat Commun 8:15556

Sabari BR, Dallagnese A, Boija A, Klein IA, Coffey EL, Shrinivas K, Abraham BJ, Hannett NM, Zamudio AV, Manteiga JC et al (2018) Coactivator condensation at super-enhancers links phase separation and gene control. Science 361:eaar3958

Schleich K, Warnken U, Fricker N, Ozturk S, Richter P, Kammerer K, Schnolzer M, Krammer PH, Lavrik IN (2012) Stoichiometry of the CD95 death-inducing signaling complex: experimental and modeling evidence for a death effector domain chain model. Mol Cell 47:306-319

Seth RB, Sun L, Ea CK, Chen ZJ (2005) Identification and characterization of MAVS, a mitochondrial antiviral signaling protein that activates NF-kappaB and IRF 3. Cell 122:669-682

Shang G, Zhang C, Chen ZJ, Bai XC, Zhang X (2019) Cryo-EM structures of STING reveal its mechanism of activation by cyclic GMP-AMP. Nature 567:389-393

Shaw AS, Chalupny J, Whitney JA, Hammond C, Amrein KE, Kavathas P, Sefton BM, Rose JK (1990) Short related sequences in the cytoplasmic domains of CD4 and CD8 mediate binding to the amino-terminal domain of the p56lck tyrosine protein kinase. Mol Cell Biol 10:1853-1862

Shen C, Yue H, Pei J, Guo X, Wang T, Quan JM (2015) Crystal structure of the death effector domains of caspase-8. Biochem Biophys Res Commun 463:297-302 
Shi J, Zhao Y, Wang Y, Gao W, Ding J, Li P, Hu L, Shao F (2014) Inflammatory caspases are innate immune receptors for intracellular LPS. Nature 514:187-192

Shin Y, Brangwynne CP (2017) Liquid phase condensation in cell physiology and disease. Science 357:eaaf4382

Su X, Ditlev JA, Hui E, Xing W, Banjade S, Okrut J, King DS, Taunton J, Rosen MK, Vale RD (2016) Phase separation of signaling molecules promotes $\mathrm{T}$ cell receptor signal transduction. Science 352:595-599

Sun L, Wu J, Du F, Chen X, Chen ZJ (2013) Cyclic GMP-AMP synthase is a cytosolic DNA sensor that activates the type I interferon pathway. Science 339:786-791

Thill PA, Weiss A, Chakraborty AK (2016) Phosphorylation of a tyrosine residue on Zap70 by Lck and its subsequent binding via an $\mathrm{SH} 2$ domain may be a key gatekeeper of $\mathrm{T}$ cell receptor signaling in vivo. Mol Cell Biol 36:2396-2402

Vajjhala PR, Lu A, Brown DL, Pang SW, Sagulenko V, Sester DP, Cridland SO, Hill JM, Schroder K, Stow JL et al (2015) The inflammasome adaptor ASC induces procaspase-8 death effector domain filaments. J Biol Chem 290:29217-29230

van Oers NS, Killeen N, Weiss A (1994) ZAP-70 is constitutively associated with tyrosine-phosphorylated TCR zeta in murine thymocytes and lymph node T cells. Immunity 1:675-685

Ve T, Vajjhala PR, Hedger A, Croll T, Dimaio F, Horsefield S, Yu X, Lavrencic P, Hassan Z, Morgan GP et al (2017) Structural basis of TIR-domain-assembly formation in MAL- and MyD88-dependent TLR4 signaling. Nat Struct Mol Biol 24:743-751

Wang L, Yang JK, Kabaleeswaran V, Rice AJ, Cruz AC, Park AY, Yin Q, Damko E, Jang SB, Raunser S et al (2010) The Fas-FADD death domain complex structure reveals the basis of DISC assembly and disease mutations. Nat Struct Mol Biol 17:13241329

Wu H, Fuxreiter M (2016) The structure and dynamics of higherorder assemblies: amyloids, signalosomes, and granules. Cell 165:1055-1066

Wu B, Peisley A, Richards C, Yao H, Zeng X, Lin C, Chu F, Walz T, Hur S (2013a) Structural basis for dsRNA recognition, filament formation, and antiviral signal activation by MDA5. Cell 152:276289

Wu J, Sun L, Chen X, Du F, Shi H, Chen C, Chen ZJ (2013b) Cyclic GMP-AMP is an endogenous second messenger in innate immune signaling by cytosolic DNA. Science 339:826-830

Wu B, Peisley A, Tetrault D, Li Z, Egelman EH, Magor KE, Walz T, Penczek PA, Hur S (2014) Molecular imprinting as a signal- activation mechanism of the viral RNA sensor RIG-I. Mol Cell 55:511-523

Xia S (2020) Biological mechanisms and therapeutic relevance of the gasdermin family. Mol Asp Med 76:

Xia S, Hollingsworth LRT, Wu H (2020) Mechanism and regulation of gasdermin-mediated cell death. Cold Spring Harb Perspect Biol 12:a036400

Xiao T, Towb P, Wasserman SA, Sprang SR (1999) Three-dimensional structure of a complex between the death domains of Pelle and Tube. Cell 99:545-555

Xu LG, Wang YY, Han KJ, Li LY, Zhai Z, Shu HB (2005) VISA is an adapter protein required for virus-triggered IFN-beta signaling. Mol Cell 19:727-740

Yang H, Wang H, Ren J, Chen Q, Chen ZJ (2017) cGAS is essential for cellular senescence. Proc Natl Acad Sci USA 114:E4612E4620

Yin Q, Tian Y, Kabaleeswaran V, Jiang X, Tu D, Eck MJ, Chen ZJ, Wu H (2012) Cyclic di-GMP sensing via the innate immune signaling protein STING. Mol Cell 46:735-745

Yin Q, Fu TM, Li J, Wu H (2015) Structural biology of innate immunity. Annu Rev Immunol 33:393-416

Zhang X, Shi H, Wu J, Zhang X, Sun L, Chen C, Chen ZJ (2013) Cyclic GMP-AMP containing mixed phosphodiester linkages is an endogenous high-affinity ligand for STING. Mol Cell 51:226235

Zhang L, Chen S, Ruan J, Wu J, Tong AB, Yin Q, Li Y, David L, Lu A, Wang WL et al (2015) Cryo-EM structure of the activated NAIP2NLRC4 inflammasome reveals nucleated polymerization. Science 350:404-409

Zhang C, Shang G, Gui X, Zhang X, Bai XC, Chen ZJ (2019) Structural basis of STING binding with and phosphorylation by TBK1. Nature 567:394-398

Zhang X, Bai XC, Chen ZJ (2020) Structures and mechanisms in the cGAS-STING innate immunity pathway. Immunity 53:43-53

Zhao Y, Yang J, Shi J, Gong YN, Lu Q, Xu H, Liu L, Shao F (2011) The NLRC4 inflammasome receptors for bacterial flagellin and type III secretion apparatus. Nature 477:596-600

Zhao B, Du F, Xu P, Shu C, Sankaran B, Bell SL, Liu M, Lei Y, Gao X, Fu $X$ et al (2019) A conserved PLPLRT/SD motif of STING mediates the recruitment and activation of TBK1. Nature 569:718-722

Zhou W, Mohr L, Maciejowski J, Kranzusch PJ (2021) cGAS phase separation inhibits TREX1-mediated DNA degradation and enhances cytosolic DNA sensing. Mol Cell 81(739-755): 